\title{
miR-17 overexpression in cystic fibrosis airway epithelial cells decreases interleukin-8 production
}

\author{
Irene K. Oglesby ${ }^{1,4}$, Sebastian F. Vencken ${ }^{1,4}$, Raman Agrawal' ${ }^{2}$, Kevin Gaughan ${ }^{1}$, \\ Kevin Molloy ${ }^{1}$, Gerard Higgins $^{3}$, Paul McNally ${ }^{3}$, Noel G. McElvaney ${ }^{1}$, \\ Marcus A. Mall ${ }^{2}$ and Catherine M. Greene ${ }^{1}$
}

\begin{abstract}
Affiliations: ${ }^{1}$ Respiratory Research Division, Department of Medicine, Royal College of Surgeons in Ireland, Education and Research Centre, Beaumont Hospital, Dublin, Ireland. ${ }^{2}$ Department of Translational Pulmonology, Translational Lung Research Center Heidelberg, Member of the German Center for Lung Research (DZL), University of Heidelberg, Heidelberg, Germany. ${ }^{3}$ National Children's Research Centre, Our Lady's Children's Hospital, Crumlin, Dublin, Ireland. "Both authors contributed equally.
\end{abstract}

Correspondence: Catherine M. Greene, Respiratory Research Division, Dept of Medicine, Royal College of Surgeons in Ireland, Education and Research Centre, Beaumont Hospital, Dublin 9, Ireland.

E-mail: cmgreenedrcsi.ie

ABSTRACT Interleukin (IL)-8 levels are higher than normal in cystic fibrosis (CF) airways, causing neutrophil infiltration and non-resolving inflammation. Overexpression of microRNAs that target IL-8 expression in airway epithelial cells may represent a therapeutic strategy for cystic fibrosis.

IL- 8 protein and mRNA were measured in cystic fibrosis and non-cystic fibrosis bronchoalveolar lavage fluid and bronchial brushings ( $\mathrm{n}=20$ per group). miRNAs decreased in the cystic fibrosis lung and predicted to target IL-8 mRNA were quantified in $\beta \mathrm{ENaC}$-transgenic, cystic fibrosis transmembrane conductance regulator (Cftr)-/- and wild-type mice, primary cystic fibrosis and non-cystic fibrosis bronchial epithelial cells and a range of cystic fibrosis versus non-cystic fibrosis airway epithelial cell lines or cells stimulated with lipopolysaccharide, Pseudomonas-conditioned medium or cystic fibrosis bronchoalveolar lavage fluid. The effect of miRNA overexpression on IL-8 protein production was measured.

miR-17 regulates IL-8 and its expression was decreased in adult cystic fibrosis bronchial brushings, $\beta E N a C$-transgenic mice and bronchial epithelial cells chronically stimulated with Pseudomonas-conditioned medium. Overexpression of miR-17 inhibited basal and agonist-induced IL-8 protein production in F508del-CFTR homozygous CFTE290 ${ }^{-}$tracheal, CFBE410 ${ }^{-}$and/or IB3 bronchial epithelial cells.

These results implicate defective CFTR, inflammation, neutrophilia and mucus overproduction in regulation of miR-17. Modulating miR-17 expression in cystic fibrosis bronchial epithelial cells may be a novel anti-inflammatory strategy for cystic fibrosis and other chronic inflammatory airway diseases.

@ERSpublications

Overexpression of miR-17 in cystic fibrosis airway epithelial cells decreases interleukin-8 protein production http://ow.ly/MZbXB

This article has supplementary material available from erj.ersjournals.com

Received: Sept 042014 | Accepted after revision: April 292015 | First published online: July 092015

Support statement: Funding for this work is gratefully acknowledged from The National Children's Research Centre (C/13/1 to CM Greene) and the Deutsche Forschungsgemeinschaft (MA2081/4-1 to MA Mall). Funding information for this article has been deposited with FundRef.

Conflict of interest: Disclosures can be found alongside the online version of this article at erj.ersjournals.com

Copyright OERS 2015 


\section{Introduction}

Cystic fibrosis (CF) is a multisystem disorder; however, its pulmonary manifestations are largely responsible for the associated high morbidity and mortality [1,2]. A striking feature of CF lung disease is the high level of infiltrating neutrophils. The large numbers of neutrophils that accumulate within the $\mathrm{CF}$ lung secrete proteases that overwhelm the normal antiprotease and antimicrobial defences, and together with neutrophil-derived oxidants cause derangement of the respiratory epithelial surface and promote pro-inflammatory gene expression [3]. Thus neutrophils and their products play a major role in pulmonary inflammation in CF. Importantly, neutrophil proteases, such as neutrophil elastase, and other neutrophil- or microbial-derived pro-inflammatory factors present in the CF lung can exacerbate neutrophil-dominated inflammation by inducing expression of interleukin (IL)-8 [4]. IL-8 is abundantly present in the CF lung and is a potent neutrophil chemokine. Thus, inhibiting IL-8 expression represents an anti-inflammatory strategy to control excessive neutrophil-dominated lung inflammation in CF.

IL-8 is expressed by bronchial epithelial cells and macrophages in the lung. CF is largely an airway disease, and the vast surface area of the potentially inflamed CF bronchial epithelium represents a major source of IL-8. Recent studies suggested that strategies designed to interfere with IL-8 gene transcription using transcription factor decoys may have therapeutic potential for CF [5, 6]. Small interfering (si)RNAmediated inhibition of IL-8 expression in polarised airway epithelial cells is another strategy [7]; however, interfering in the post-transcriptional regulation of IL- 8 is also possible.

Micro (mi)RNAs) are short endogenous non-coding single-stranded RNAs that function as post-transcriptional negative regulatory molecules. They modulate target gene expression via target mRNA degradation and/or translational repression and are implicated as key regulators in almost all biological processes $[8,9]$. miRNAs bind to miRNA recognition elements (MREs) largely located in the $3^{\prime}$ untranslated region (UTR) of target mRNAs. Therapeutic modulation of miRNA levels is possible by overexpression using synthetic miRNA mimics or antisense inhibition by anti-miRs [10]. In the CF lung, where IL-8 expression is persistently elevated, we speculate that miRNAs regulating IL-8 expression may be reciprocally decreased. If so an overexpression approach using miRNA mimics may have therapeutic potential.

The first in vivo CF miRNA profiling studies were carried out using CF versus non-CF bronchial brushings [11]; however the role of miRNA in modulating IL-8 was not addressed at that point. Hu et al. [12] have demonstrated that IL- 8 is susceptible to modulation by miR-520b in breast cancer cells, while Yu et al. [13] have shown that the miR-17/20 cluster regulates the IL-8 $3^{\prime} \mathrm{UTR}$. Other studies have reported that miR-203 and miR-93/106b regulate IL-8 expression [14, 15]. BHATtacharyya et al. [16] reported how increased levels of miR-155 indirectly elevate IL-8 in CF IB3 cells, CF bronchial brushings and CF neutrophils. More recently, FABBRI et al. [17] showed that miR-93 regulates IL-8 production in CF airway epithelial cell lines in response to heat-killed Pseudomonas aeruginosa.

Here we quantify IL-8 mRNA and protein expression levels in clinical samples from the lungs of people with and without CF. We investigate the contribution of the altered miRNA expression profile in CF bronchial brushings to the higher than normal IL-8 levels and explore reasons for the reduced levels of miR-17, a validated regulator of IL-8. We compare the findings to candidate miRNA expression in $\beta \mathrm{ENaC}$-transgenic mice with $\mathrm{CF}$-like lung disease and $\mathrm{CF}$ transmembrance conductance regulator $(\mathrm{Cftr})$-/mice, and explore the potential to decrease basal and agonist-induced IL- 8 expression by CF bronchial epithelial cells using miRNA mimics.

\section{Methods and materials}

In silico analysis

Bioinformatic analysis was performed using the miRNA target prediction databases TargetScanHuman 6.2, MicroRNA.org, PITA, and MicroCosm to search for miRNAs predicted to target the $3^{\prime}$ UTR sequence of human IL-8 or the $3^{\prime}$ UTR of murine KC/CXCL1.

\section{Bronchoalveolar lavage fluid and bronchial brush sampling, miRNA profiling, primary epithelial cell culture and quantitative reverse transcriptase PCR}

Following written informed consent according to a protocol approved by the Beaumont Hospital ethics review board (Dublin, Ireland): 1) bronchoalveolar lavage (BAL) fluid was recovered from CF patients $(\mathrm{n}=12$, mean \pm SD age $27 \pm 1.4$ years, male:female $4: 8)$ and non-CF control individuals $(n=12$, age $60 \pm 4.8$ years, male:female 3:9), as previously described [18]; and 2) bronchial brushings were sampled and RNA isolated as previously described [11] from CF ( $\mathrm{n}=8$, age 28.2 \pm 4.6 years, male:female 4:4) and non-CF control individuals ( $\mathrm{n}=8$, age $48.5 \pm 6.02$ years, male:female $4: 4)$. CF individuals were confirmed by sweat testing and/ or genotyping and are listed in online supplementary table S1. Non-CF control individuals were undergoing exploratory bronchoscopy in the investigation into idiopathic cough. Five of the adult $\mathrm{CF}$ and non-CF 
bronchial brush samples were previously used for miRNA expression profiling [11]; the remainder were used for subsequent miRNA validation studies and quantitative reverse transcriptase (RT)-PCR.

Following written informed consent according to a protocol approved by the ethics review board at Our Lady's Children's Hospital Crumlin (Dublin, Ireland), primary epithelial cultures were established from seven CF (age 3 \pm 0.61 years, male:female 5:2, $\Delta$ F508/ $\Delta$ F508 $(n=5), \Delta F 508 / C .1766+1 G>A(n=1)$ and $\Delta F 508 /$ unknown $(\mathrm{n}=1)$ ) and two non-CF (age $0.54 \pm 0.45$ years, male:female 2:0) bronchial brushings collected as part of the Study of Host Immunity and Early Lung Disease in CF (SHIELD CF), as previously described [19]. In addition, mRNA expression profiling data was used from three $\Delta F 508 / \Delta F 508$ and three non-CF bronchial brushings previously reported [20].

\section{IL-8 ELISA}

IL-8 protein concentrations in BAL fluid and cell supernatants were determined by sandwich ELISA (R\&D Systems, Abingdon, UK).

Measurement of mRNA expression levels by quantitative RT-PCR

Total RNA was extracted using TRIzol (Invitrogen, OR, USA) and reverse transcribed into cDNA using Quantitect Reverse Transcription Kit (Qiagen, Valencia, CA, USA). mRNA expression was measured using LightCycler 480 SYBR Green I Master Mix (Roche, Burgess Hill, UK) on the Roche LC480 Lightcycler. Expression of IL- 8 mRNA relative to $\beta$-actin was determined using the $2^{-\Delta \Delta C t}$ method. Primers used were as follows. IL-8: forward 5'-TTTTGCCAAGG AGTGCTAAAGA-3', reverse 5'-AACCCTCTGCACCCAGTT TTC-3'; and $\beta$-actin: forward 5'-GGACTTCG AGCAAGAGATGG-3' reverse $5^{\prime}$-AGGAAGGAAGGCRTGG AAGAG-3'. Quantitative RT-PCR was also performed for STAT3, TIMP2 and p21 using the following primers. Signal transducer and activator of transcription (STAT)-3: forward 5'-GGACATCAGCGGTA AGACCC-3', reverse 5'-GCTCTCTGGCCGACA ATACT-3'; tissue inhibitor of metalloproteinase (TIMP)2: forward 5'-CTCATTGCAGGAAAGGCCGA-3' reverse 5'-GGAGGAGATGTAGCACGGGA-3'; and p21: forward 5'-GTGGACCTGTCACTGTCTTGT-3' reverse 5'-GGTAGAAATCTGTCATGCTGGTCT-3'. All quantitative RT-PCR experiments were performed in triplicate and included no-template controls.

\section{Measurement of human miRNA levels}

cDNA was generated from total RNA using TaqMan MicroRNA Reverse Transcription kits (Applied Biosystems, Foster City, CA, USA). miRNA expression was measured on the Roche LC480 Lightcycler using TaqMan assays (Applied Biosystems). Expression of miRNAs relative to U6 small nuclear (sn)RNA was determined using the $2^{-\Delta \Delta C t}$ method. Quantitative RT-PCR experiments in cell lines were performed in triplicate, a minimum of three times and included no-template controls.

\section{Mouse studies}

Experimental animals were housed in a specific pathogen-free animal facility at the University of Heidelberg (Heidelberg, Germany) and had free access to chow and water. The $\beta \mathrm{ENaC}$-transgenic ( $\beta \mathrm{ENaC}-\mathrm{Tg}$ ) mouse was originally generated on a mixed genetic background $(\mathrm{C} 3 \mathrm{H} / \mathrm{He} \times \mathrm{C} 57 \mathrm{BL} / 6)$ and was backcrossed to the C57BL/6 background as described previously [21]. Transgene-positive mice were identified by PCR of genomic DNA [22]. For transcript analyses, we collected whole-lung homogenates from 2-week-old mice and trachea and main stem bronchi from 6-week-old mice, as previously described [23], i.e. at ages when $\beta E N a C-T g$ mice were reported to exhibit CF-like lung disease [24]. Gut corrected Cftr knockout (Cftr-/-) mice overexpressing human CFTR in the intestine under control of the fatty acid binding protein promoter were kindly provided by Jeffrey A. Whitsett (Division of Pulmonary Biology, Cincinnati Children's Hospital Medical Center, Cincinnati, OH, USA), backcrossed to the C57BL/6 background and genotyped as previously described $[21,25]$. Transcript analyses in Cftr-/- mice were performed in bronchi of 30-week-old mice, i.e. at an age when Cftr-/- mice were reported to exhibit multiorgan pathology [26]. Total RNA was isolated using TRIzol reagent. Real-time quantitative RT-PCR for mmu-miR-17 and mmu-let-7b normalised to U6 snRNA was performed using TaqMan assays. BAL was performed in 6-week-old $\beta \mathrm{ENaC}$-Tg mice and 30-week-old Cftr-/- mice, and differential cell counts were determined from cytospin preparations, as previously described [24]. In all experiments, age-matched wild-type littermates served as controls.

\section{Luciferase reporter assays}

HEK293 cells from the European Collection of Cell Cultures (Public Health England, Porton Down, UK) $\left(2 \times 10^{5}\right.$ in triplicate) were transiently co-transfected for $24 \mathrm{~h}$ with an IL-8 $3^{\prime}$ UTR firefly luciferase reporter vector containing the full-length 3'UTR (250 ng) (kindly provided by R. Pestell, Thomas Jefferson University, Philadelphia, PA, USA [13]), a constitutive Renilla luciferase vector (100 ng) and $30 \mathrm{nM}$ synthetic Pre-miR miRNA Precursors (PM) or AntimiR miRNA Inhibitors (AM) (Applied Biosystems), as indicated or with a scrambled control. Transfections were performed using GeneJuice (Novagen, Madison,WI, USA) for plasmid 
DNA and RiboJuice (Novagen) for miRNA in OptiMEM reduced serum media (Life Technologies), as recommended. AntimiRs do not always degrade their target miRNA and we measured variable antimiR-specific target miRNA knockdown of $20-80 \%$ using transfection conditions that were optimised previously to ensure uniform transfection across all wells [27]. Lysates were prepared $36 \mathrm{~h}$ post-transfection and luciferase activities were measured using the Luciferase Assay System (Promega) and coelenterazine (Marker Gene Technologies, Eugene, OR, USA). Relative luciferase activity was calculated.

\section{HBE140-/CFBE410-, 9HTEo-/CFTE290- and S9/IB3 epithelial cell studies}

The human non-CF $16 \mathrm{HBE} 14 \mathrm{o}^{-}$and $9 \mathrm{HTEo}^{-}$and F508del homozygous CFBE41o ${ }^{-}$and CFTE29o bronchial and tracheal epithelial cell lines were supplied as a gift from D. Gruenert (California Pacific Medical Centre Research Institute, San Francisco, CA, USA) [28, 29]. IB3 cells are a CF bronchial epithelial cell line (F508del/W1282X), S9s are their isogenic non-CF counterpart and both were obtained from Pamela Zeitlin (Johns Hopkins Children's Centre, Baltimore, MD, USA) [30]. 16HBE14o ${ }^{-} / \mathrm{CFBE}_{10}{ }^{-}$and $9 \mathrm{HTEO}^{-} / \mathrm{CFTE} 29 \mathrm{o}^{-}$cells were grown in MEM+GlutaMax (Gibco) with $10 \%$ fetal calf serum (FCS; Gibco) and $1 \%$ penicillin-streptomycin (Pen-Strep; Gibco). 9HTEo ${ }^{-} / \mathrm{CFTE} 29 \mathrm{o}^{-}$cells were supplemented with $1 \times$ nonessential amino acids (Lonza). IB3 and S9 cells were grown in LHC-8 medium (Gibco) with 5\% FCS and $1 \%$ Pen-Strep. In some experiments S9 cells were grown to a density of $5 \times 10^{5} \mathrm{cells} \cdot \mathrm{cm}^{-2}$ and chronically stimulated (5 days) [31] with $1 \%$ Pseudomonas-conditioned medium (PCM) [27] in LHC-8 medium (Gibco) with 1\% FCS and 1\% Pen-Strep. Media were changed every day.

Cells were seeded at $1 \times 10^{5}, 0.5 \times 10^{5}$ and $0.8 \times 10^{5}$ cells $\cdot \mathrm{cm}^{-2}$ for $16 \mathrm{HBE} 14 \mathrm{o}^{-} / \mathrm{CFBE} 41 \mathrm{o}^{-}$, 9HTEo-/ $\mathrm{CFTE}_{29 \mathrm{o}^{-}}$and S9/IB3, respectively and were transfected with $30 \mathrm{nM}$ scrambled control or PMs using RiboJuice transfection reagent. In initial experiments, cells were co-transfected with a fluorescently labelled non-targeting miRIDIAN miRNA mimic (Dharmacon) to monitor and optimise transfection efficiency; $>80-90 \%$ of cells were transfected as monitored by epifluorescence and miRNA expression was increased following PM transfection [24]. Mature miRNA expression was measured in transfected cells to ensure efficient transfection. 24- or 48-h post-transfection cells were left unstimulated or stimulated with P. aeruginosa lipopolysaccharide (LPS) $\left(10 \mu \mathrm{g} \cdot \mathrm{mL}^{-1}\right.$; Sigma-Aldrich, Kilkenny, Ireland), $1 \%$ PCM or $10 \mu \mathrm{L} \cdot \mathrm{mL}^{-1} \mathrm{CF}$ BAL fluid for a further $24 \mathrm{~h}$. Supernatants were retained for IL-8 protein analysis.

\section{Statistical analysis}

All analyses were performed using GraphPad PRISM 4.0 (San Diego, CA, USA). Results are expressed as mean \pm SEM and were compared by t-test or ANOVA, as indicated. For ANOVA, the post hoc Dunnett test was used for pair-wise comparison. Differences were considered significant at $p \leqslant 0.05$.

\section{Results}

Levels of IL-8 are increased in CF versus non-CF BSL fluid and bronchial brushings

Many studies have reported higher than normal levels of IL-8 in the CF lung. In order to confirm this observation in our patient cohort, levels of IL-8 protein were measured in BAL fluid collected from subjects with and without CF. Figure 1a shows significantly higher levels of IL-8 in CF versus non-CF BAL fluid samples.

Next, IL-8 mRNA expression was measured in a selection of CF and non-CF bronchial brushings. Figure $1 \mathrm{~b}$ shows that expression of IL- 8 mRNA, relative to $\beta$-actin mRNA, is significantly increased in CF in vivo.
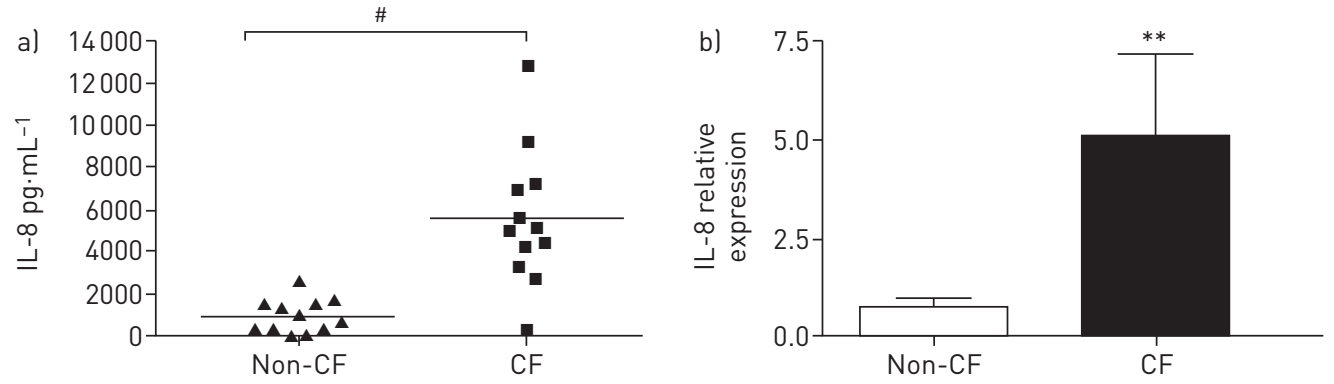

FIGURE 1 Interleukin (IL)-8 levels in cystic fibrosis (CF) and non-CF bronchoalveolar lavage fluid (BALF) and bronchial brushings. a) IL-8 protein levels were measured using ELISA in BALF from 12 CF and 12 non-CF subjects; b) relative expression of IL-8 mRNA in bronchial brushings (CF $n=8$, non-CF $n=8$ ) measured using quantitative reverse transcriptase PCR. Data are presented as mean \pm SEM and were compared by t-test. ${ }^{* *}$ : $p<0.01 ;{ }^{\#}: p<0.0001$. 


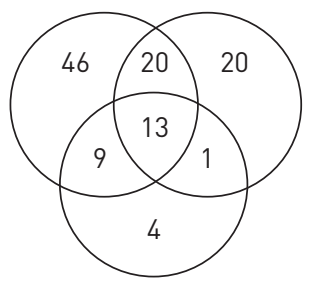

PITA and MicroCosm targets

b)

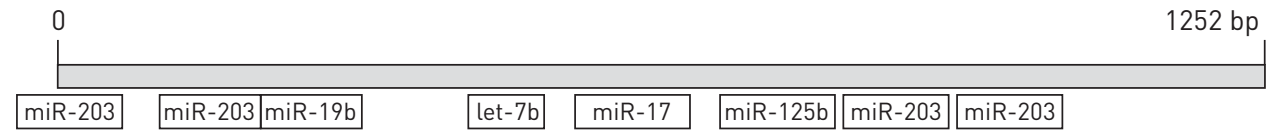

FIGURE 2 Micro (mi)RNAs predicted to target interleukin (IL)-8 in cystic fibrosis (CF) bronchial epithelium. a) In silico analysis of miRNAs predicted to regulate IL-8. The miRNA target prediction databases TargetScan version 6.2, MicroRNA.org. PITA and MicroCosm Targets were used to identify predicted miRNA sites in the full-length 1252-nucleotide human IL-8 mRNA (NM_000584). b) Binding sites of selected miRNAs in the IL-8 3 ' untranslated region predicted by TargetScan 6.2 and microRNA.org.

miRNAs predicted to target the IL-8 3'UTR in CF bronchial epithelium

A selection of miRNA target prediction databases was interrogated to identify miRNAs with a possible role in regulation of IL-8. Figure 2a shows the number of miRNAs predicted to target the IL-8 $3^{\prime} \mathrm{UTR}$ by TargetScan 6.2, MicroRNA.org and PITA/MicroCosm. These lists were cross-compared with miRNAs known to be decreased in vivo in CF bronchial brushings [11]. Table 1 shows details of miRNAs selected for further study on the basis of 1) the relative expression level (significant or not) of the miRNA in CF versus non-CF bronchial epithelium; 2) the number of predicted MREs in the IL-8 3'UTR; and 3) the miRNA support vector recognition (miR-SVR) score. The selected miRNAs were let-7b, miR-17 and miR-203, which are all significantly decreased in vivo in CF bronchial brushings, and miR-19b and miR-125b, which are predicted to target IL-8, but the expression of which is unaltered between CF and non-CF brushings. Of the selected miRNAs, levels of let-7b and miR-17 were independently validated by miRNA assay as being lower than normal in the remaining original CF versus non-CF bronchial brush samples used in the profiling (miR-17: $1 \pm 0.42$ versus $0.38 \pm 0.21$, let- $7 \mathrm{~b}$ : $1 \pm 0.85$ versus $0.57 \pm 0.21$ for control versus $\mathrm{CF}$ bronchial brushings, respectively; not significant). Figure $2 \mathrm{~b}$ depicts the full-length human IL- 8 3'UTR with predicted binding locations for the selected miRNAs of interest.

miR-17 is the most effective regulator of an IL-8 3'UTR luciferase reporter

In order to determine which of the chosen miRNAs regulate expression of IL-8, HEK293 cells were transiently transfected with an IL-8 3'UTR luciferase reporter vector. Co-transfection with PMs showed that PM-let-7b and PM-17 significantly decreased luciferase gene expression compared to a scrambled control (fig. 3a). PM-203, PM-19b and PM-125b did not decrease luciferase expression, so these miRNAs were not explored further.

Luciferase assays were repeated using AMs to knockdown the expression of let-7b and miR-17. Under identical transfection conditions AM-17, but not AM-let-7b, significantly increased luciferase expression

\begin{tabular}{lcccc}
\multicolumn{2}{l}{ TABLE 1 } & Micro (mi)RNAs selected for further study & & \\
miRNA & MREs in IL-8 3'UTR $\mathbf{n}$ & miR-SVR score & Expression in vivo in CF & p-value \\
\hline let-7b & 1 & -0.0209 & 0.47 & $<0.001$ \\
miR-17 & 1 & -1.1807 & 0.56 & $<0.001$ \\
miR-203 & 4 & $-0.071,-0.015,-0.9735^{+}$ & 0.53 & 0.003 \\
miR-125b & 1 & -0.1553 & 0.91 & NS \\
miR-19b & 1 & -0.463 & 0.74 & NS
\end{tabular}

MRE: miRNA recognition elements; IL: interleukin; UTR: untranslated region; miR-SVR: miRNA support vector recognition; CF: cystic fibrosis; NS: nonsignificant. \#: predicted by microRNA.org $<<-0.1$ considered good). ๆ: relative expression in CF versus non-CF bronchial brushings; ${ }^{+}$: miR-SVR score not predicted by microRNA.org for one MRE. 
(fig. 3b). miR-221 is not predicted to target IL-8 and AM-221 did not decrease luciferase activity. These data indicate that miR-17 is the most effective regulator of IL-8 from the miRNAs decreased in vivo in CF versus non-CF bronchial brushings.

\section{Expression of miR-17 target genes in CF versus non-CF bronchial brushings}

In addition to IL-8, other targets of miR-17 include signal transducer and activator of transcription (STAT) 3, TIMP2, p21, fibronectin and FoxA1 [32-36]. mRNA array data from CF and non-CF bronchial brushings were analysed to determine whether the expression of these genes is increased in CF [20]. Online supplementary figure $\mathrm{S} 1$ shows that while there was no increase in expression of fibronectin or FoxA1, there was a trend towards increased levels of STAT3, TIMP2, p21 and IL-8 transcripts in the CF samples. However, due to the small sample size and/or the possibility that miR-17 may affect only the protein rather than both the mRNA and protein levels of these targets, differences were not statistically significant.

\section{Expression of mmu-miR-17 in airway tissues from $\beta E N a C$-transgenic mice and $\mathrm{Cftr}-/$ - mice} miR-17 and let-7b are conserved in mammals. In order to determine whether expression of these miRNAs is altered in mice with CF-like lung disease, the levels of mmu-miR-17 and mmu-let-7b were measured in whole-lung homogenates and native airway tissues (trachea and mainstem bronchi) from 2-week-old and 6-week-old $\beta \mathrm{ENaC}$-Tg mice and wild-type littermates and normalised to U6 snRNA. As in the human CF bronchial brushings, the expression of mmu-miR-17 was significantly decreased in the airway tissues of the $\beta \mathrm{ENaC}-\mathrm{Tg}$ mice at 2 and 6 weeks (fig. 4a). Mmu-let-7b expression tended to be decreased in $\beta \mathrm{ENaC}$-Tg compared to wild-type mice; however, this difference did not reach statistical significance.

We also quantified miR-17 and let-7b levels in Cftr-/- mice compared to wild-type littermates. There was no significant difference in let-7b expression. Surprisingly miR-17 levels were higher in Cftr-/- versus wild-type mice (fig. $4 \mathrm{~b}$ ). In contrast to $\beta \mathrm{ENaC}$-Tg mice which develop a robust spontaneous neutrophilic inflammation, we did not detect increased neutrophil counts in the lungs of Cftr-/- mice (online supplementary fig. S2). Furthermore, Cftr-/- mice, unlike $\beta E N a C-T g$ mice, do not develop goblet cell metaplasia and airway mucus obstruction [24, 37]. These results suggest that factors associated with chronic airways disease may contribute to reduced miR-17 levels in CF-like lung disease in vivo, and may be responsible for the observed differences in miR-17 levels in $\beta \mathrm{ENaC}-\mathrm{Tg}$ versus $\mathrm{Cftr}$-/- mice.

\section{Expression of miR-17 in CF and non-CF cell lines, primary cells and under chronic inflammatory conditions}

miR-17 levels were decreased in vivo in $\mathrm{CF}$ bronchial brushings and in $\beta \mathrm{ENaC}-\mathrm{Tg}$ mice with spontaneous airway neutrophilia and mucus obstruction. Next we quantified its levels in a selection of CF and non-CF airway epithelial cells lines and primary bronchial cells from children with and without CF. We did not observe a consistent difference in miR-17 levels across all of the CF versus non-CF cell lines (fig. 5); miR-17 levels were slightly or significantly decreased in IB3 and CFBE cells versus their non-CF counterparts, respectively, whereas miR-17 was significantly increased in CFTE versus HTE cells. The CFTE effect may be cell line- or trachea-specific. In contrast to the adult bronchial brushings, we did not
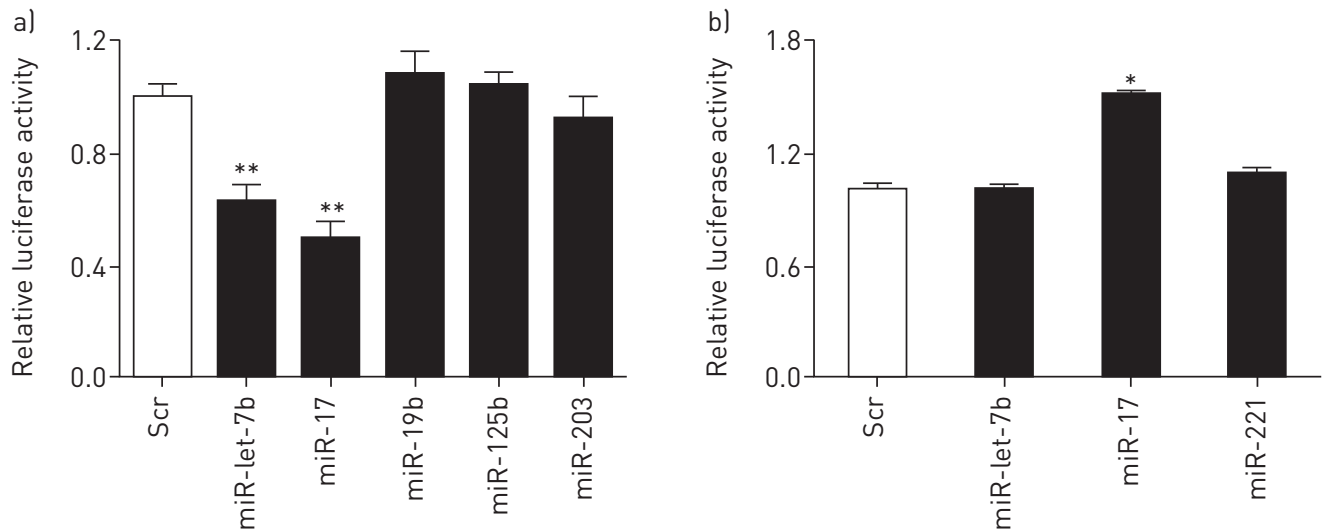

FIGURE 3 miR-17 regulates interleukin (IL)-8. Relative luciferase activity in HEK293 cells $\left(1 \times 10^{5}\right.$ in triplicate) transiently transfected with IL-8 $3^{\prime}$ untranslated region (pGL3-IL-8-3'UTR) and a constitutive Renilla luciferase reporter plasmid (pRLSV40) and co-transfected with al synthetic pre-miR or b) anti-miR for miRNAs as indicated. Firefly luciferase activity was normalised to the Renilla luciferase activity. Data are presented as mean \pm SEM and were compared by t-test. Scr: scrambled control. $n=3 .{ }^{*}: p<0.05 ;{ }^{* *}: p<0.01$. 

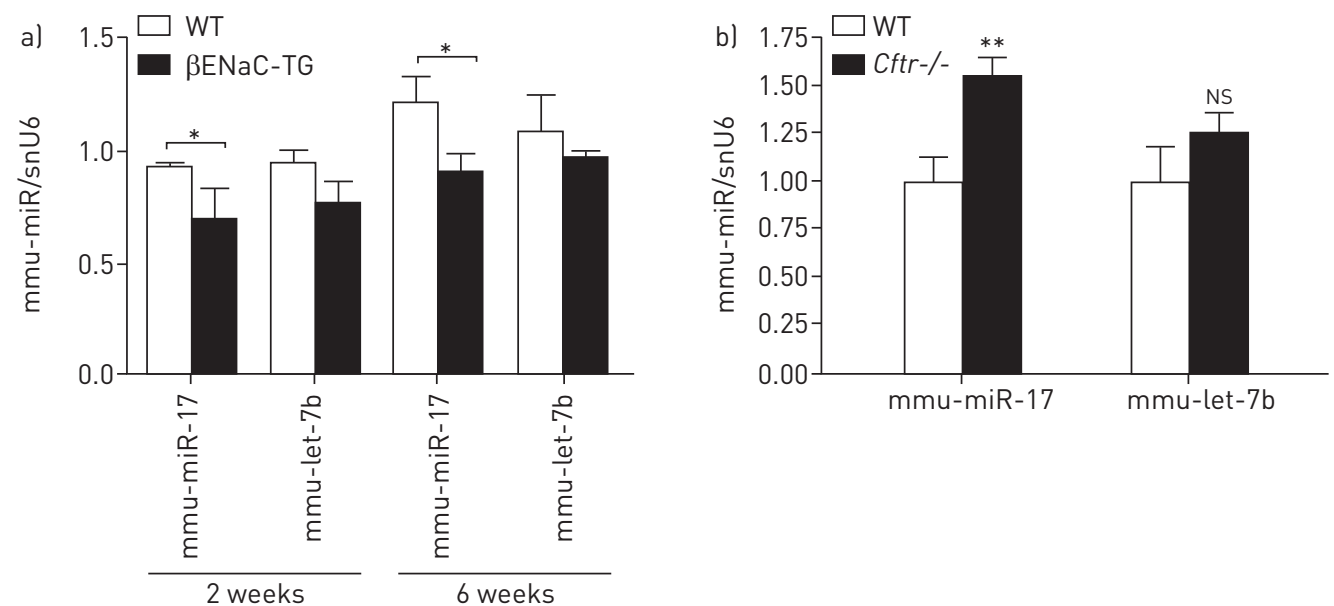

FIGURE 4 mmu-miR-17 expression in $\beta E N a C$-Tg and Cftr-/- versus wild-type (WT) mice. Relative expression of murine miR-17 and let-7b was determined by quantitative reverse transcriptase PCR using individual TaqMan murine miRNA assays and normalised to U6 small nuclear (sn)RNA in whole-lung homogenates of a) 2-week-old ( $n=5)$ and trachea and bronchi of 6 -week-old ( $n=6$ of each) wild-type and $\beta E N a C$-Tg mice or b) bronchi of 30 -week-old ( $n=6$ each) wild-type and Cftr-/- mice. Data are presented as mean \pm SEM and were compared by t-test. Ns: nonsignificant. *: $p<0.05 ;{ }^{* *}: p<0.01$.

observe a significant decrease in miR-17 levels in primary CF cells from children, although miR-17 levels showed a trend towards reduction in age-matched non-CF primary cells. While this may be an age-specific effect, it is also possible that the effect may be due to a lack of specific microbial- and host-derived pro-inflammatory factors in the paediatric CF lung samples, and/or removal of these stimuli in the process of establishing the primary cell cultures. To test this we examined miR-17 expression in S9 cells that underwent chronic (5-day) stimulation with Pseudomonas-conditioned medium. Figure 5 shows that miR-17 expression is significantly decreased under these conditions.

\section{Pre-miR-17 inhibits basal and LPS-induced IL-8 protein production in CF bronchial and tracheal} epithelial cells

In order to test whether miR-17 overexpression can inhibit basal or stimulus-induced IL-8 expression in human CF airway epithelial cells, CFBE410 ${ }^{-}$and CFTE290 ${ }^{-}$cells were transfected with PM-17 and treated with $10 \mu \mathrm{g} \cdot \mathrm{mL}^{-1}$ LPS to induce IL-8 expression, or left untreated. mRNA levels of IL-8 and other miR-17 gene targets were quantified in the scrambled control versus the PM-17-transfected cells (online

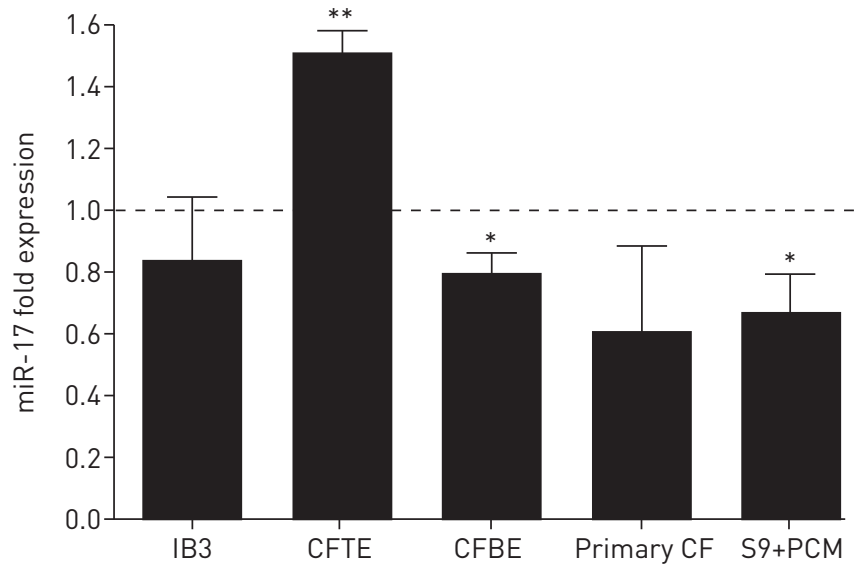

FIGURE 5 miR-17 in cell lines, primary cells and under chronic inflammatory conditions. Relative expression of miR-17 was determined by quantitative reverse transcriptase PCR using TaqMan miRNA assays and normalised to U6 small nuclear (sn)RNA in IB3 (n=6), CFTE290- $(n=3)$ and CFBE410 $(n=3)$ epithelial cell lines, cystic fibrosis (CF) paediatric primary epithelial cultures $(n=7)$ and S9 cells stimulated for 5 days with $1 \%$ Pseudomonas-conditioned medium (PCM; $\mathrm{n}=5)$. Data are presented as fold change \pm SEM compared to S9 $(n=8)$, 9HTEo- $(n=3), 16$ HBE140- $(n=3)$, non-CF primary epithelial cells $(n=2)$ or untreated S9 cells $(n=5)$, respectively (i.e. dotted line at one-fold). Data were compared by t-test. *: $p<0.05 ;{ }^{* *}$ : $p<0.01$. 
supplementary fig. S1B); only IL-8 was decreased in LPS-stimulated CFTEs transfected with PM-17. Figure 6 shows that compared to PM-let-7b, which had no significant effect on basal or LPS-induced IL-8 protein production, overexpression of miR-17 significantly decreased basal $(62.5 \%$ and $46.5 \%)$ and LPS-induced IL-8 protein production $(44.6 \%$ and $43.7 \%)$ in the CFBE41o ${ }^{-}$and CFTE29o ${ }^{-}$cell lines, respectively. In the tracheal but not bronchial cells PM-let-7b significantly increased basal IL- 8 protein production; this may be a cell line- or tracheal-specific effect.

Pre-miR-17 inhibits agonist-induced IL-8 protein production in IB3 CF bronchial epithelial cells Finally, the effect of PM-17 on IL-8 protein production induced by LPS and other pro-inflammatory agonists representative of the CF lung was evaluated in the CF airway epithelial line IB3, as this cell line was previously used for CF miRNA studies by BHATTACHARYya et al. [16]. Similar to treatment with LPS, stimulation of IB3 cells with PCM or CF BAL fluid for $24 \mathrm{~h}$ led to significant increases in IL-8 protein production (fig. 7). Although PM-17 had no effect on basal IL-8 protein production in these cells, it significantly inhibited agonist-induced IL-8 protein production in all cases.

\section{Discussion}

miR-17 post-transcriptionally regulates IL-8 expression. Here we show that overexpression of miR-17 can interfere with basal and CF lung-specific agonist-induced IL-8 protein production from CF airway epithelial cells in vitro, suggesting that a pre-miR-17-based medicine could have potential to treat the inflammatory manifestations of CF lung disease.

miRNA profiling identified miRNAs that are altered in vivo in CF bronchial brushings [11]. Of the miRNAs that were significantly decreased in the CF samples a number were predicted to target the IL- 8 3'UTR. Taking into account their miR-SVR scores, three miRNAs were chosen for this study: let-7b, miR-17 and miR-203. IL-8 is a validated target of these and other miRNAs [12-15]. Notwithstanding that miR-203 levels were lower than normal in CF bronchial brushings and that there are four putative miR-203 MREs in the IL-8 3'UTR, PM-203 was ineffective in the luciferase reporter gene studies, therefore it was not explored further. Instead, a comparison between miR-17 and let-7b was performed by knocking them down and determining if this could enhance expression of the IL-8 $3^{\prime}$ UTR luciferase reporter gene. Based on these studies miR-17 was found to be more effective than let-7b at regulating IL-8. This observation was substantiated in the CF airway epithelial cell studies whereby PM-17, but not PM-let-7b, significantly impaired basal and agonist-induced IL-8 protein production.

miR-17 is encoded on the miR-17 92 gene cluster on chromosome 13. This cluster has a role in tissue development and miR-17 is known to be important in lung epithelial bud morphogenesis [38] and proliferation of lung epithelial progenitor cells [39, 40]. Largely overexpressed in lung cancers [41], there are few reports of underexpression of miR-17 in the lung, with the recent exception of pulmonary fibrosis and asthma [42, 43]. FABBRI et al. [17] have identified miR-93 as a miRNA that regulates IL-8 expression and that may be implicated in CF pathology. Interestingly, miR-93 and miR-17 are also part of the same

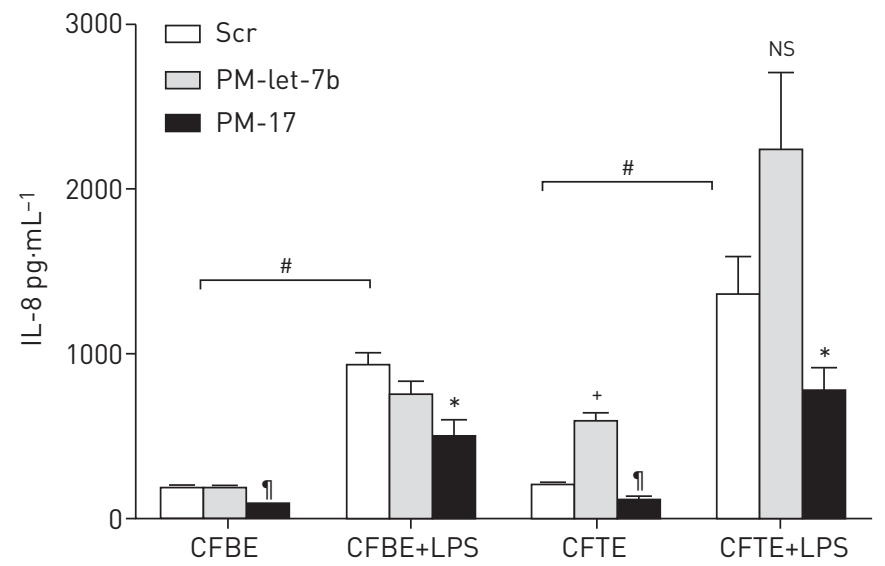

FIGURE 6 Effects of micro (mi)RNA overexpression on interleukin (IL)-8 protein production in CFBE410- and CFTE290- cells stimulated with lipopolysaccharide (LPS). IL-8 (pg. $\left.\mathrm{mL}^{-1}\right)$ was measured by ELISA in the supernatant of cells $\left(1 \times 10^{5}\right.$ cells $\left.\mathrm{mL}^{-1}\right)$ transfected with a synthetic pre-miR (PM) for let-7b and miR-17 or a scrambled control $(\mathrm{Scr})$, and stimulated with LPS $(10 \mu \mathrm{g})$ for $24 \mathrm{~h}$. Data are presented as mean \pm SEM and were compared to untreated $\left(^{\#}: p<0.0001\right)$ or scrambled control $\left(*: p<0.05 ;\right.$ ?: $\left.p<0.005 ;{ }^{+}: p<0.0001\right)$ by t-test. NS: nonsignificant. 


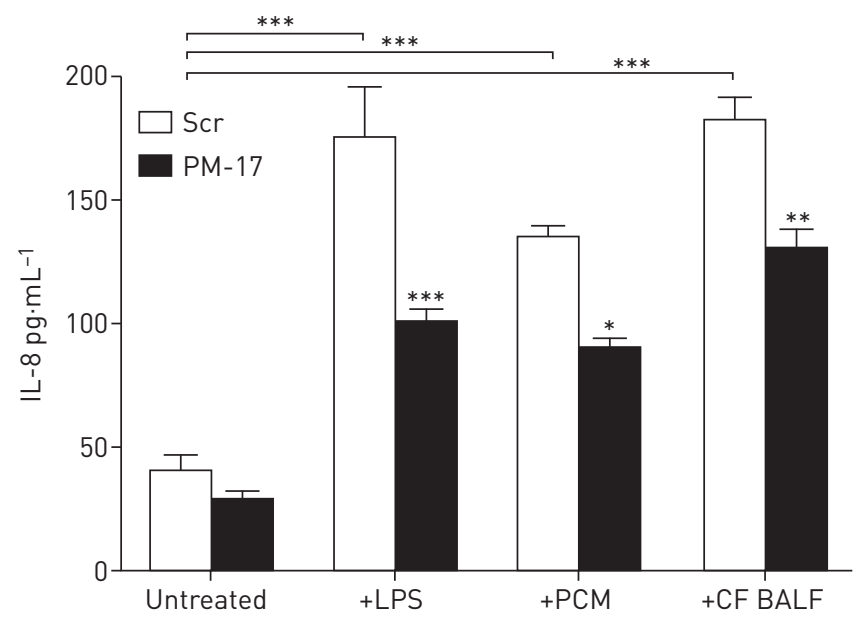

FIGURE 7 Effects of micro (mi)RNA overexpression on interleukin (IL)-8 protein production in IB3 cells stimulated with lipopolysaccharide (LPS), Pseudomonas-conditioned medium (PCM) or cystic fibrosis (CF) bronchoalveolar lavage fluid (BALF). IL-8 ( $\left.\mathrm{pg} \cdot \mathrm{mL}^{-1}\right)$ was measured by ELISA in the supernatant of cells $\left(1 \times 10^{5}\right.$ cells $\left.\cdot \mathrm{mL}^{-1}\right)$ transfected with a synthetic pre-miR for miR-17 (PM-17) or a scrambled control (Scr) and stimulated with LPS $(10 \mu \mathrm{g}), 1 \%$ PCM or $10 \mu \mathrm{L}$ CF BALF for $24 \mathrm{~h}(\mathrm{n}=3)$. Data are presented as mean \pm SEM and were compared by ANOVA. ${ }^{*}: p<0.05 ;{ }^{* *}: p<0.01 ;{ }^{* * *}: p<0.001$.

miRNA gene family (the miR-17 microRNA precursor family), but unlike miR-17, miR-93 is encoded on chromosome 7 . We previously reported that miR-93* is increased rather than decreased in CF versus non-CF bronchial brushings [11]; however there are no data to suggest that miR-93* regulates IL-8 expression. In addition to IL-8, other targets of miR-17 include STAT3, TIMP2, p21, fibronectin and FoxA1 [32-36]. We examined the expression of these mRNAs in bronchial brushings and CF airway epithelial cells transfected with PM-17; however there were no major changes in the levels of these transcripts, except for IL-8, in the prospectively collected CF bronchial brushings or LPS-stimulated CFTE cells transfected with PM-17.

In the lungs of $\beta \mathrm{ENaC}-\mathrm{Tg}$ mice, expression of miR-17 was decreased compared to their wild-type counterparts. In contrast, miR-17 was increased rather than decreased in Cftr-/-versus wild-type mice. In the context of CF, altered miRNA expression in the airways could occur in response to increased chemokine production, airway neutrophilia or mucus obstruction, among others. To the best of our knowledge, in Cftr-/- mice the hyperinflammatory response with robust lung neutrophilia and increased chemokine production is only observed when mice are challenged with Pseudomonas or LPS. In the absence of such challenges, these mice have not been reported to develop spontaneous CF-like neutrophilic airways disease with increased CXCL1 and CXCL2 levels and mucus plugging, but have been shown to develop alveolar abnormalities with parenchymal interstitial thickening and recruitment of macrophages in the alveoli that were most pronounced in older mice on the C57BL6 background [26, 44]. For this reason, we used aged (30-week-old) Cftr-/- mice. Consistent with previous reports of alveolar infiltration with macrophages, we found that BAL macrophages were elevated in the Cftr-/- mice versus wild-type controls (Supplementary Fig 2B). However, we did not detect spontaneous neutrophilia in the Cftr-/- mice. While a subtle increase in neutrophils was reported [45], this was not observed in other mice with loss of Cftr, albeit on a different genetic background [46]. In contrast, $\beta E N a C$-Tg mice develop robust spontaneous neutrophilic inflammation in addition to CF-like airways mucus obstruction [22, 24, 37]. Furthermore, Cftr-/- mice, unlike $\beta E N a C-T g$ mice, do not develop goblet cell metaplasia and airway mucus obstruction [24,37]; however the CF mouse model used in the current study is on a gut-corrected background, which may change the overall inflammatory phenotype. Mucus obstruction and the lack of spontaneous airway neutrophilia and inflammation are key differences between the two models that may be responsible for the observed differences in miR-17 levels. Interestingly, inflammation in the mouse lung is controlled differently than in humans. Mice do not express IL-8, instead they express a number of functional homologues of IL- 8 with KC/CXCL1 considered to be the closest. Murine CXCL2, CXCL5 and CCL2 also share overlapping functions with IL-8. None of these transcripts or KC/CXCL1 is predicted to be regulated by mmu-miR-17 or mmu-let-7b.

We were initially surprised that miR-17 levels were not decreased in CF versus non-CF primary airway epithelial cells, but given that these cells were of paediatric origin they are less likely to have been exposed to as many chronic inflammatory stimuli as the cells in the adult CF bronchial brushings. Further removal 
of any such stimuli in the process of establishing the primary cell cultures, or an age-related effect, may also account for our observations. In order to test the pro-inflammatory theory we subjected airway epithelial cells to chronic stimulation with Pseudomonas-conditioned medium. These conditions significantly decreased miR-17 expression and go some way towards explaining the mechanism by which miR-17 is decreased in the adult CF lung. Taken together, the data from the basal and agonist-stimulated cell lines, paediatric primary bronchial epithelial cells, adult bronchial brushings, and $\beta \mathrm{ENaC}-\mathrm{Tg}$ and Cftr-/- mice indicate that defective CFTR, the pro-inflammatory milieu of the CF lung, neutrophilia, goblet cell metaplasia and mucus obstruction are all factors that could contribute to altered miR-17 expression. The data do not demonstrate that decreased miR-17 expression in vivo in the CF lung is solely responsible for increased IL-8 expression.

CF lung therapeutics are targeted at liquefying mucus, decreasing inflammation, killing infectious microbes and restoring CFTR-mediated chloride ion conductance. A number of anti-inflammatory approaches indirectly target IL-8 production, for example corticosteroids, nonsteroidal anti-inflammatory drugs and possibly macrolides. In addition to IL-8, leukotriene B4 (LTB4) and other less abundant chemotactic peptides are also present in the CF lung. Strategies targeting LTB4 in CF were unsuccessful in the past $[47,48]$. That strategy not only inhibited neutrophil chemotaxis but was also cytotoxic against neutrophils, thereby killing whichever neutrophils managed to migrate to the lung in response to other chemokines. The goal in controlling the excessive neutrophil infiltration into the CF lung is to restore chemotaxis to normal rather than sub-normal levels. In this regard we aim to inhibit abnormal expression of IL- 8 while leaving intact the normal processes that are necessary and sufficient for physiological neutrophil infiltration into the lung.

miR-medicines are attractive therapeutic candidates [10]. Unlike DNA-based approaches, which require nuclear delivery, miRNAs only need to be delivered to the cytoplasm, and may be more benign to cells in terms of eliciting innate immune responses. Nonetheless, challenges exist with respect to their delivery and efficacy. We have demonstrated that it is possible to deliver functional PMs into CF bronchial epithelial cells using polyethyleneimine nanoparticles [49]. A similar strategy could be used to deliver miRNA mimics that target IL-8. Examining off-target effects of any such approach will be necessary in order to determine the specificity of the inhibition observed. Thus, nanomedicines designed to increase levels of underexpressed endogenous miRNAs represent a new alternative to the existing therapeutic strategies to treat the pulmonary manifestations of cystic fibrosis. Our results highlight miR-17 as a potential candidate for more in-depth drug development studies for CF and potentially other chronic neutrophilic lung diseases.

\section{References}

$1 \quad$ Rowe SM, Miller S, Sorscher EJ. Cystic fibrosis. N Engl J Med 2005; 352: 1992-2001.

2 Mall MA, Hartl D. CFTR: cystic fibrosis and beyond. Eur Respir J 2014; 44: 1042-1054.

3 Kelly E, Greene CM, McElvaney NG. Targeting neutrophil elastase in cystic fibrosis. Expert Opin Ther Targets 2008; 12: 145-157.

4 Nakamura H, Yoshimura K, McElvaney NG, et al. Neutrophil elastase in respiratory epithelial lining fluid of individuals with cystic fibrosis induces interleukin-8 gene expression in a human bronchial epithelial cell line. J Clin Invest 1992; 89: 1478-1484.

5 Bezzerri V, Borgatti M, Nicolis E, et al. Transcription factor oligodeoxynucleotides to NF- $\kappa \mathrm{B}$ inhibit transcription of IL-8 in bronchial cells. Am J Respir Cell Mol Biol 2008; 39: 86-96.

6 Gambari R, Borgatti M, Bezzerri V, et al. Decoy oligodeoxyribonucleotides and peptide nucleic acids-DNA chimeras targeting nuclear factor kappa-B: inhibition of IL-8 gene expression in cystic fibrosis cells infected with Pseudomonas aeruginosa. Biochem Pharmacol 2010; 80: 1887-1894.

7 Ramachandran S, Krishnamurthy S, Jacobi AM, et al. Efficient delivery of RNA interference oligonucleotides to polarized airway epithelia in vitro. Am J Physiol Lung Cell Mol Physiol 2013; 305: L23-L32.

8 Guo H, Ingolia NT, Weissman JS, et al. Mammalian microRNAs predominantly act to decrease target mRNA levels. Nature 2010; 466: 835-840.

9 Friedman R, Farh KK, Burge CB, et al. Most mammalian mRNAs are conserved targets of microRNAs. Genome Res 2009; 19: 92-105.

10 Hassan T, McKiernan PJ, McElvaney NG, et al. Therapeutic modulation of miRNA for the treatment of proinflammatory lung diseases. Expert Rev Anti Infect Ther 2012; 10: 359-368.

11 Oglesby IK, Bray IM, Chotirmall SH, et al. miR-126 is downregulated in cystic fibrosis airway epithelial cells and regulates TOM1 expression. J Immunol 2010; 184: 1702-1709.

$12 \mathrm{Hu} \mathrm{N}$, Zhang J, Cui W, et al. miR-520b regulates migration of breast cancer cells by targeting hepatitis $\mathrm{B}$ X-interacting protein and interleukin-8. J Biol Chem 2011; 286: 13714-13722.

$13 \mathrm{Yu} \mathrm{Z,} \mathrm{Willmarth} \mathrm{NE,} \mathrm{Zhou} \mathrm{J,} \mathrm{et} \mathrm{al.} \mathrm{microRNA} \mathrm{17/20} \mathrm{inhibits} \mathrm{cellular} \mathrm{invasion} \mathrm{and} \mathrm{tumor} \mathrm{metastasis} \mathrm{in} \mathrm{breast}$ cancer by heterotypic signaling. Proc Natl Acad Sci USA 2010; 107: 8231-8236.

14 Wei $\mathrm{T}, \mathrm{Xu} \mathrm{N}$, Meisgen F, et al. Interleukin- 8 is regulated by miR-203 at the posttranscriptional level in primary human keratinocytes. Eur J Dermatol 2013 [In press DOI: 10.1684/ejd.2013.1997].

15 Chuang TD, Luo X, Panda H, et al. miR-93/106b and their host gene, MCM7, are differentially expressed in leiomyomas and functionally target F3 and IL-8. Mol Endocrinol 2012; 26: 1028-1042.

16 Bhattacharyya S, Balakathiresan NS, Dalgard C, et al. Elevated miR-155 promotes inflammation in cystic fibrosis by driving hyperexpression of interleukin-8. J Biol Chem 2011; 286: 11604-11615.

17 Fabbri E, Borgatti M, Montagner G, et al. Expression of microRNA-93 and interleukin-8 during Pseudomonas aeruginosa-mediated induction of proinflammatory responses. Am J Respir Cell Mol Biol 2014; 50: 1144-1155. 

MyD88/IRAK/TRAF-6 in human bronchial epithelium. J Biol Chem 2001; 276: 35494-35499.

19 Verrière V, Higgins G, Al-Alawi M, et al. Lipoxin A4 stimulates calcium-activated chloride currents and increases airway surface liquid height in normal and cystic fibrosis airway epithelia. PLoS One 2012; 7: e37746.

20 McKiernan PJ, Molloy K, Cryan SA, et al. Long noncoding RNA are aberrantly expressed in vivo in the cystic fibrosis bronchial epithelium. Int J Biochem Cell Biol 2014; 52: 184-191.

21 Johannesson B, Hirtz S, Schatterny J, et al. CFTR regulates early pathogenesis of chronic obstructive lung disease in $\beta E N a C$-overexpressing mice. PLoS One 2012; 7: e44059.

22 Mall M, Grubb BR, Harkema JR, et al. Increased airway epithelial $\mathrm{Na}^{+}$absorption produces cystic fibrosis-like lung disease in mice. Nat Med 2004; 10: 487-493.

23 Anagnostopoulou P, Dai L, Schatterny J, et al. Allergic airway inflammation induces a pro-secretory epithelial ion transport phenotype in mice. Eur Respir J 2010; 36: 1436-1447.

24 Mall MA, Harkema JR, Trojanek JB, et al. Development of chronic bronchitis and emphysema in $\beta$-epithelial $\mathrm{Na}^{+}$ channel-overexpressing mice. Am J Respir Crit Care Med 2008; 177: 730-742.

25 Zhou L, Dey CR, Wert SE, et al. Correction of lethal intestinal defect in a mouse model of cystic fibrosis by human CFTR. Science 1994; 266: 1705-1708.

26 Durie PR, Kent G, Phillips MJ, et al. Characteristic multiorgan pathology of cystic fibrosis in a long-living cystic fibrosis transmembrane regulator knockout murine model. Am J Pathol 2004; 164: 1481-1493.

27 Oglesby IK, Chotirmall SH, McElvaney NG, et al. Regulation of cystic fibrosis transmembrane conductance regulator by microRNA-145, -223 , and -494 is altered in $\Delta$ F508 cystic fibrosis airway epithelium. J Immunol 2013; 190: 3354-3362.

28 Bruscia E, Sangiuolo F, Sinibaldi P, et al. Isolation of CF cell lines corrected at $\triangle$ F508-CFTR locus by SFHR-mediated targeting. Gene Ther 2002; 9: 683-685.

29 Kunzelmann K, Schwiebert EM, Zeitlin PL, et al. An immortalized cystic fibrosis tracheal epithelial cell line homozygous for the $\Delta$ F508 CFTR mutation. Am J Respir Cell Mol Biol 1993; 8: 522-529.

30 Zeitlin PL, Lu L, Rhim J, et al. A cystic fibrosis bronchial epithelial cell line: immortalization by adeno-12-SV40 infection. Am J Respir Cell Mol Biol 1991; 4: 313-319.

31 Strickertsson JA, Rasmussen LJ, Friis-Hansen L. Enterococcus faecalis infection and reactive oxygen species down-regulates the miR-17-92 cluster in gastric adenocarcinoma cell culture. Genes (Basel) 2014; 5: 726-738.

32 Zhang M, Liu Q, Mi S, et al. Both miR-17-5p and miR-20a alleviate suppressive potential of myeloid-derived suppressor cells by modulating STAT3 expression. J Immunol 2011; 186: 4716-4724.

33 Li SH, Guo J, Wu J, et al. miR-17 targets tissue inhibitor of metalloproteinase 1 and 2 to modulate cardiac matrix remodeling. FASEB J 2013; 27: 4254-4265.

34 Wang M, Gu H, Qian $\mathrm{H}$, et al. miR-17-5p/20a are important markers for gastric cancer and murine double minute 2 participates in their functional regulation. Eur J Cancer 2013; 49: 2010-2021.

35 Shan SW, Lee DY, Deng Z, et al. MicroRNA MiR-17 retards tissue growth and represses fibronectin expression. Nat Cell Biol 2009; 11: 1031-1038.

$36 \mathrm{Xu} \mathrm{Z}$, Zhang $\mathrm{C}$, Cheng L, et al. The microRNA miR-17 regulates lung FoxA1 expression during lipopolysaccharide-induced acute lung injury. Biochem Biophys Res Commun 2014; 445: 48-53.

37 Zhou Z, Duerr J, Johannesson B, et al. The ENaC-overexpressing mouse as a model of cystic fibrosis lung disease. J Cyst Fibros 2011; 10: S172-S182.

38 Carraro G, El-Hashash A, Guidolin D, et al. miR-17 family of microRNAs controls FGF10-mediated embryonic lung epithelial branching morphogenesis through MAPK14 and STAT3 regulation of E-Cadherin distribution. Dev Biol 2009; 333: 238-250.

39 Lu Y, Thomson JM, Wong HY, et al. Transgenic over-expression of the microRNA miR-17-92 cluster promotes proliferation and inhibits differentiation of lung epithelial progenitor cells. Dev Biol 2007; 310: 442-453.

40 Lu Y, Okubo T, Rawlins E, et al. Epithelial progenitor cells of the embryonic lung and the role of microRNAs in their proliferation. Proc Am Thorac Soc 2008; 5: 300-304.

41 Hayashita Y, Osada H, Tatematsu Y, et al. A polycistronic microRNA cluster, miR-17-92, is overexpressed in human lung cancers and enhances cell proliferation. Cancer Res 2005; 65: 9628-9632.

42 Dakhlallah D, Batte K, Wang Y, et al. Epigenetic regulation of miR-17 92 contributes to the pathogenesis of pulmonary fibrosis. Am J Respir Crit Care Med 2013; 187: 397-405.

43 Martinez-Nunez RT, Bondanese VP, Louafi F, et al. A microRNA network dysregulated in asthma controls IL-6 production in bronchial epithelial cells. PLoS One 2014; 9: e111659.

44 Kent G, Iles R, Bear CE, et al. Lung disease in mice with cystic fibrosis. J Clin Invest 1997; 100: 3060-3069.

45 Legssyer R, Huaux F, Lebacq J, et al. Azithromycin reduces spontaneous and induced inflammation in $\Delta \mathrm{F} 508$ cystic fibrosis mice. Respir Res 2006; 7: 134.

46 Livraghi-Butrico A, Kelly EJ, Wilkinson KJ, et al. Loss of Cftr function exacerbates the phenotype of $\mathrm{Na}^{+}$ hyperabsorption in murine airways. Am J Physiol Lung Cell Mol Physiol 2013; 304: L469-L480.

47 Fayon M. CF - emerging therapies: modulation inflammation. Paediatr Respir Rev 2006; 7: S170-S174.

48 Lee E, Lindo T, Jackson N, et al. Reversal of human neutrophil survival by leukotriene B(4) receptor blockade and 5-lipoxygenase and 5-lipoxygenase activating protein inhibitors. Am J Respir Crit Care Med 1999; 160: 2079-2085.

49 McKiernan PJ, Cunningham O, Greene CM, et al. Targeting miRNA-based medicines to cystic fibrosis airway epithelial cells using nanotechnology. Int J Nanomedicine 2013; 8: 3907-3915 